Supporting Information

\title{
LABEL-FREE CANCER STEM-LIKE CELL ASSAY CONDUCTED AT A SINGLE CELL LEVEL USING MICROFLUIDIC MECHANOTYPING DEVICES
}

\author{
Miyu Terada ${ }^{\dagger}$, Sachiko Ide ${ }^{\dagger}$, Toyohiro Naito ${ }^{\dagger}$, Niko Kimura $^{\dagger}$, Michiya Matsusaki ${ }^{\ddagger}$, \\ Noritada Kaji ${ }^{* *}$ \\ 'Department of Applied Chemistry, Graduate School of Engineering, Kyushu University, 744 Motooka, \\ Nishi-ku, Fukuoka 819-0395, Japan \\ Department of Applied Chemistry, Graduate School of Engineering, Osaka University, 2-1 Yamadaoka, \\ Suita, Osaka 565-0871, Japan \\ *Corresponding author: kaji@cstf.kyushu-u.ac.jp
}




\section{TABLE OF CONTENTS}

Figure S1. The cell sizes measured via direct observation....S-3

Figure S2. Influence of the ALDH activity assay in HT29 cells....S-4

Figure S3. The gating processes in the ALDH activity assay of HT 29 cells using an inhibitor, DEAB....S-

5

Figure S4. The gating processes in the ALDH activity assay of Caco-2 cells using an inhibitor, DEAB....S6

Figure S5. The gating processes in the ALDH activity assay of HeLa cells using an inhibitor, DEAB...S-7 Figure S6. The gating processes in the ALDH activity assay of MDA-MB-231 cells using an inhibitor, DEAB....S-8

Figure S7. Live cell collection after the ALDH activity assay for the following proliferation and viability assays....S-9

Figure S8. The proliferation assay after FACS operation....S-11

Table S1. Electrical conductivity of the buffer and medium....S-5

Table S2. The viability assay after FACS operation....S-12 


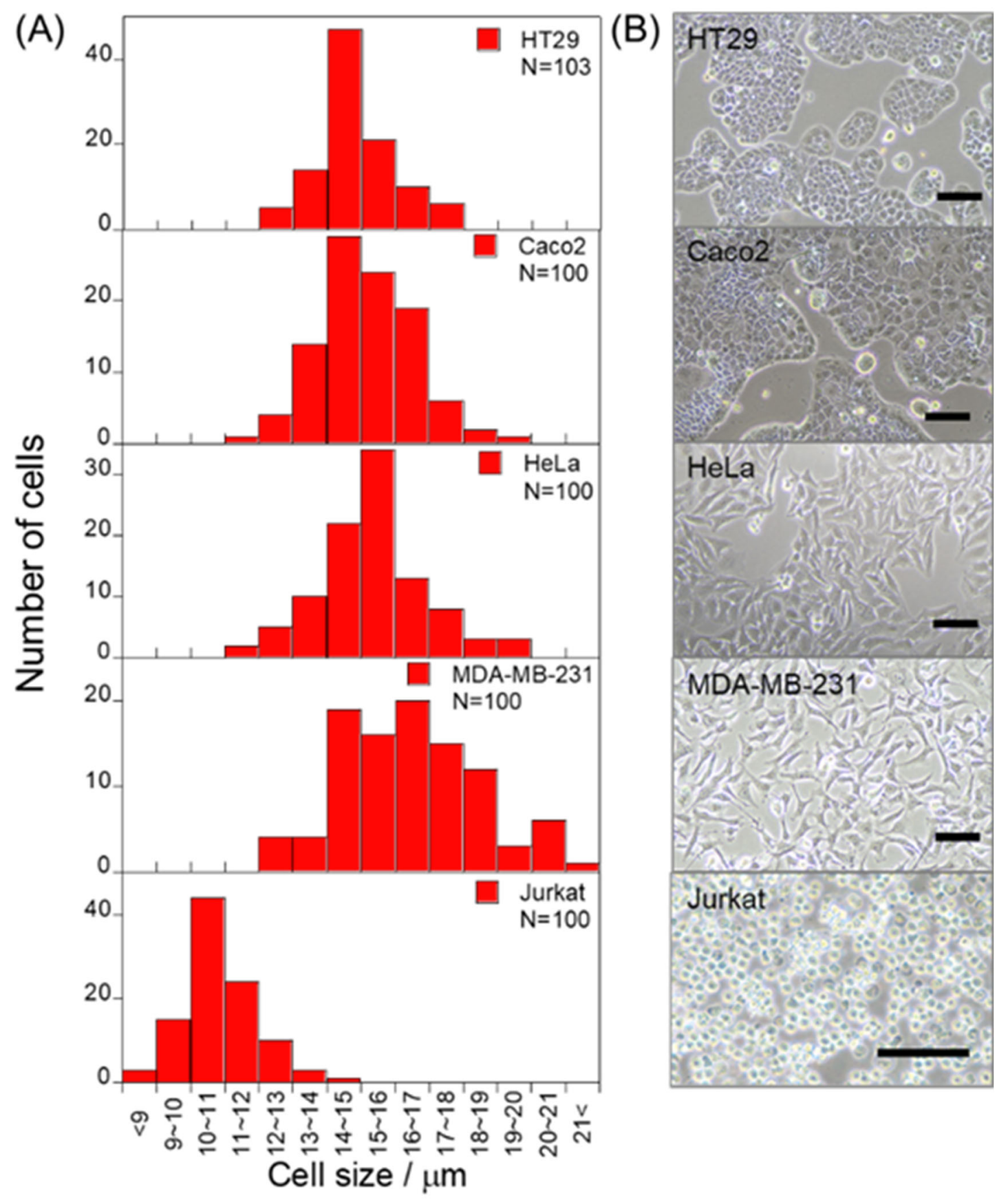

Figure S1. Cell diameter measurements obtained via direct observation. The diameter of all cells, except Jurkat cells, was measured in a suspended state immediately after subjection to trypsin treatment. Data were excluded from the measurements if the cell shapes were not evidently spherical or appeared as aggregates. (A) Histograms of the diameter of HT29, Caco-2, HeLa, MDA-MB-231, and Jurkat cells, arranged from top to bottom. (B) Typical cultured cell images in culture flasks before subjection to trypsin treatment. All scale bars are $100 \mu \mathrm{m}$. 


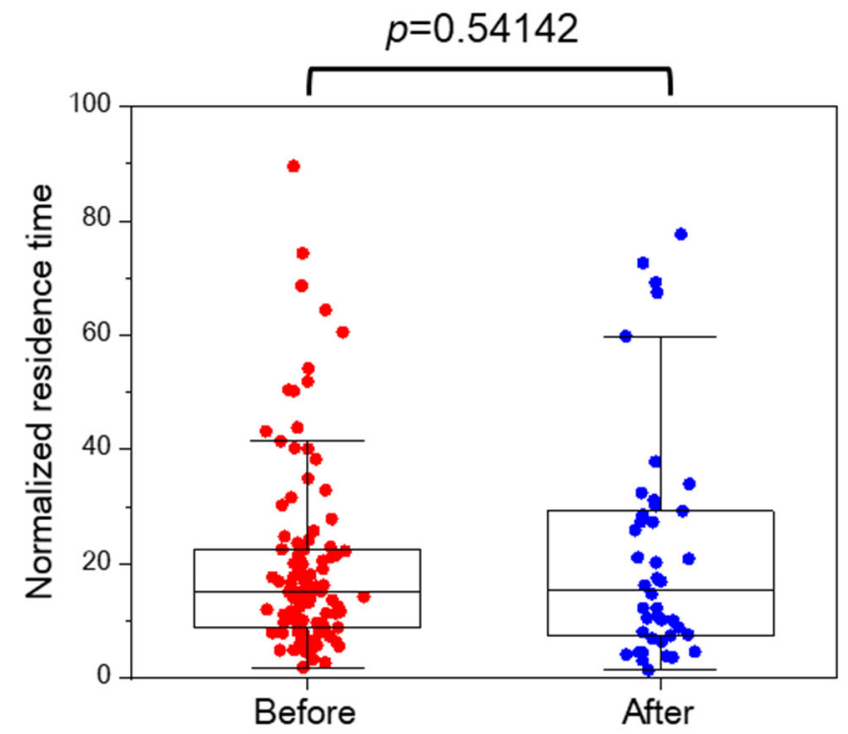

Figure S2. Normalized residence time of HT29 cells before and after the ALDH activity assay indicated as red and blue dots, respectively. In the ALDH activity assay, the fluorogenic substrate, AldeRed ${ }^{\mathrm{TM}}$ 588-A, was added to the cells and was allowed to diffuse freely into intact and viable cells. The acid formed, as a representative of the fluorescence produced via ALDH activity, remained trapped inside the cells, enabling flow cytometry analysis and FACS. The $p$ value indicates that there are no significant differences in cell deformability between the cells before and after the ALDH activity assay. The number of measured cells was $n=103$ and $n=42$ before and after the ALDH activity assay, respectively. 
(A)

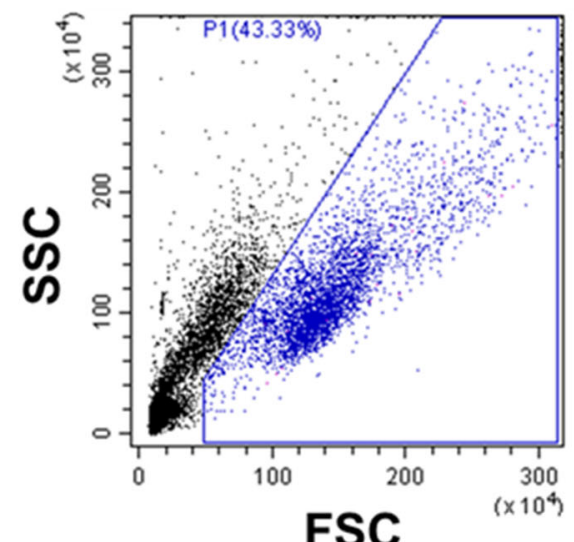

(C)

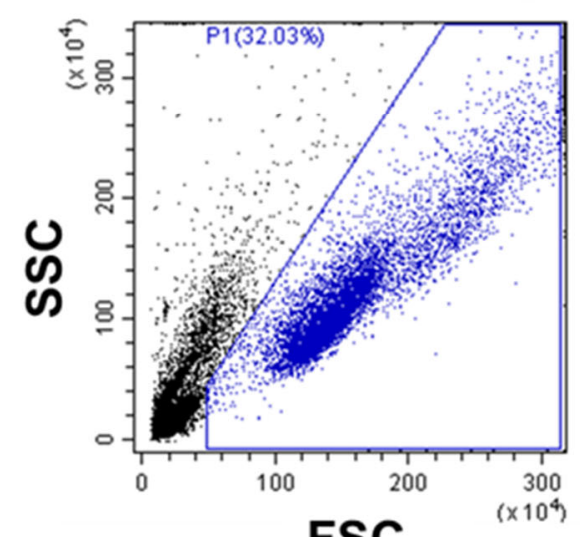

FSC
(B)

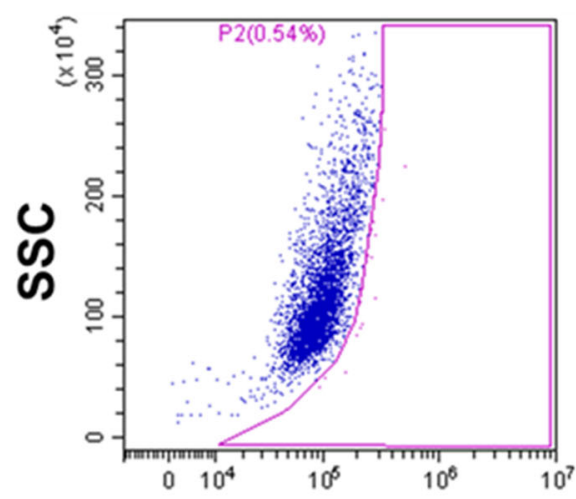

AldeRed 588-A

(D)

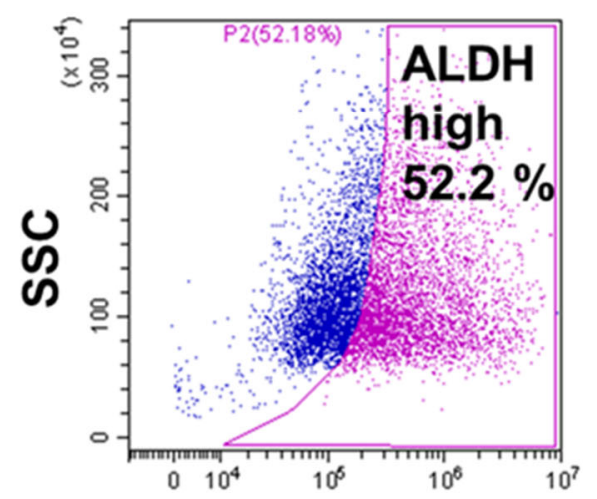

AldeRed 588-A

Figure S3. (A, B) and (C, D) show the results of flow cytometry analysis of HT29 cells based on the ALDH activity assay with and without a selective inhibitor of ALDH, N,N-diethylaminobenzaldehyde (DEAB), respectively. Based on the intensities of forward-scattered light (FSC) and side-scattered light (SSC) shown in Figures (A) and (C), where FSC intensity is proportional to the diameter of the cell and SSC intensity, enabling obtainment of discernment information on cell internal structure such as granule density and nuclear shape, the gates were placed and the following fluorescence intensity analyses were performed for the gated cell population depicted by using blue dots in Figures (A) and (C). Figures (B) and (D) show the fluorescence intensity of AldeRed ${ }^{\mathrm{TM}}$ 588-A with and without DEAB, respectively. Almost half of the cells showed high ALDH activity as depicted by using purple dots in the region gated by considering the results depicted in (B). 
(A)

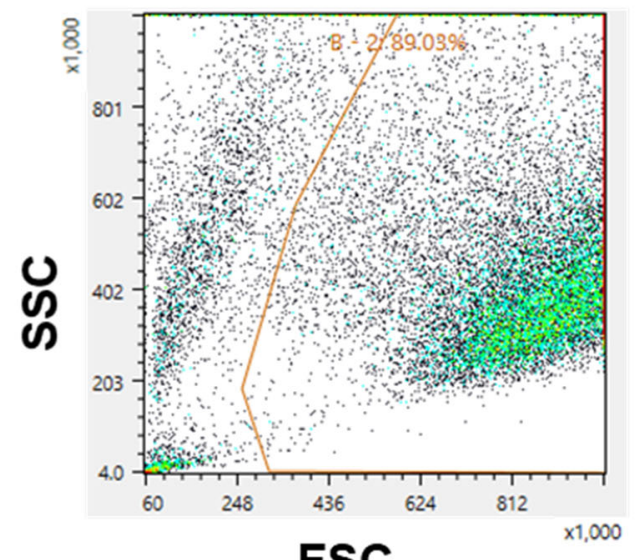

FSC

(C)

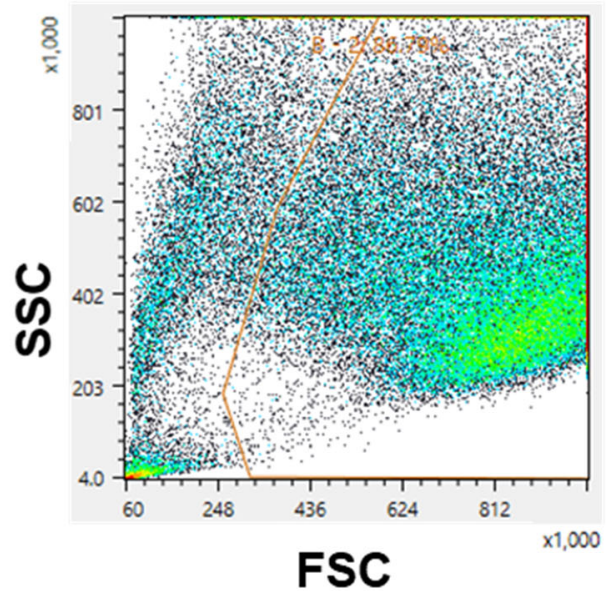

(B)

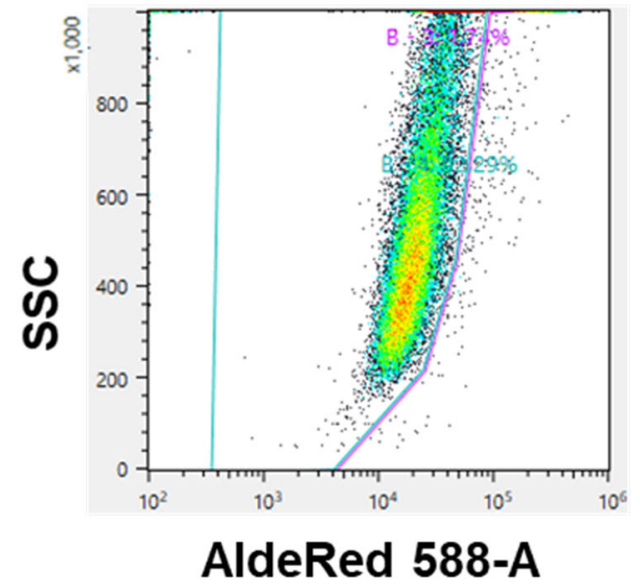

(D)

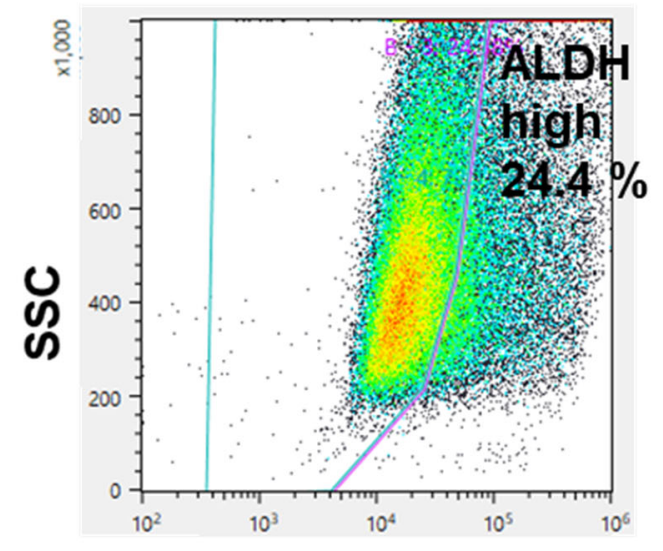

AldeRed 588-A

Figure S4. (A, B) and (C, D) show the results of flow cytometry analysis of Caco-2 cells based on the ALDH activity assay with and without DEAB, respectively. Based on the intensities of FSC and SSC shown in Figures (A) and (C), the gates were placed and the following fluorescence intensity analyses were performed for the gated cell population indicated by using orange lines in Figures (A) and (C). Figures (B) and (D) show the fluorescence intensity of AldeRed ${ }^{\mathrm{TM}}$ 588-A with and without DEAB, respectively. Approximately a quarter of the cells showed high ALDH activity as indicated by using purple lines in the region gated by considering the results depicted in (B). 
(A)

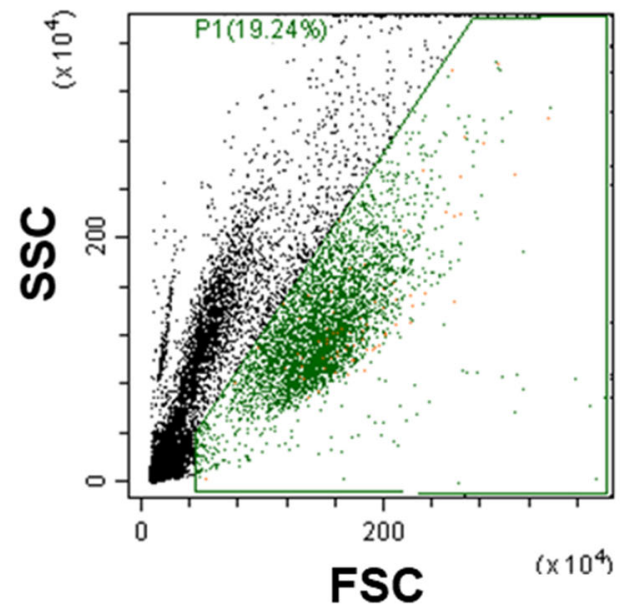

(C)

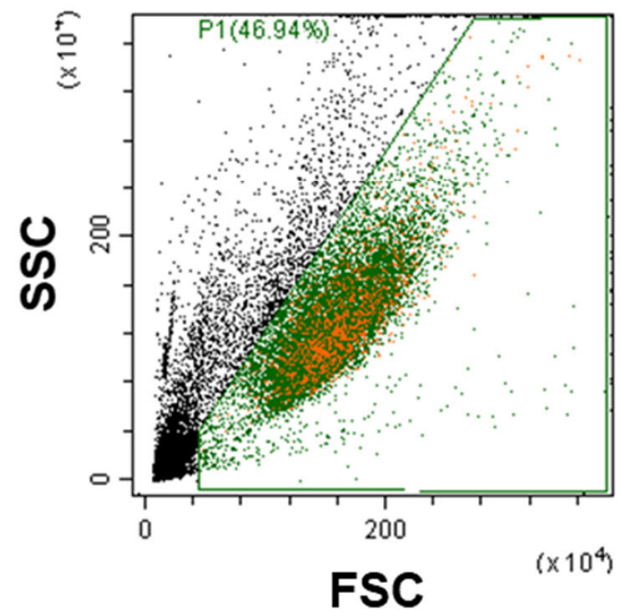

(B)

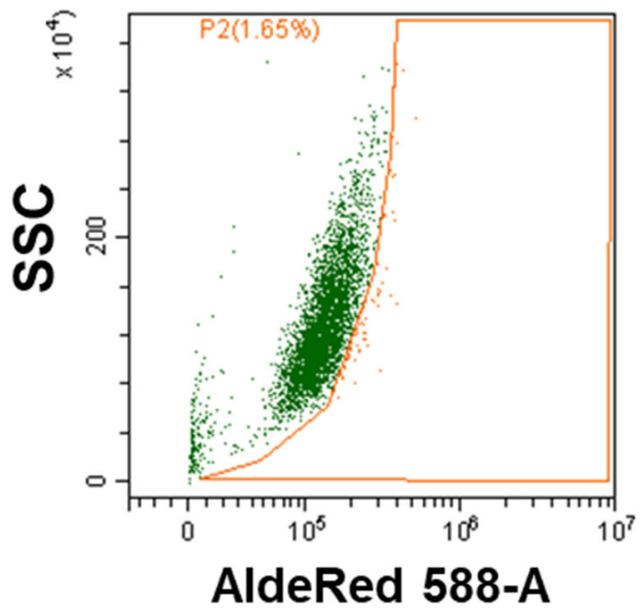

(D)

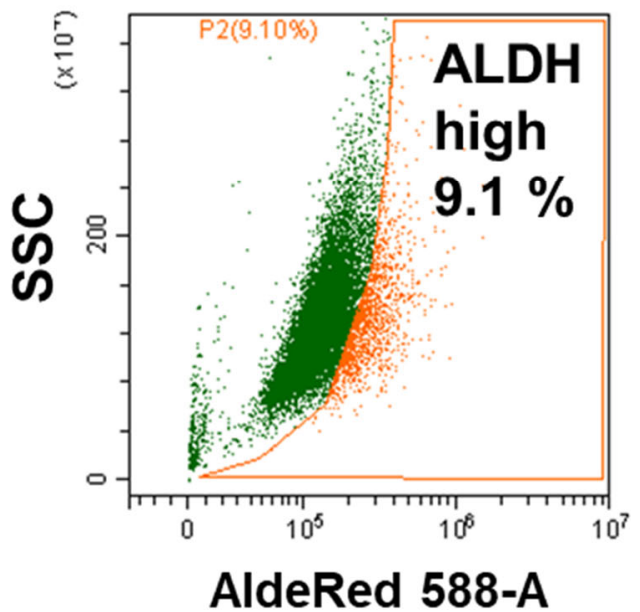

Figure S5. (A, B) and (C, D) show the results of flow cytometry analysis of HeLa cells based on the ALDH activity assay with and without DEAB, respectively. Based on the intensities of FSC and SSC shown in Figures (A) and (C), the gates were placed and the following fluorescence intensity analyses were performed for the gated cell population as indicated by using green lines in Figures (A) and (C). Figures (B) and (D) show the fluorescence intensity of AldeRed ${ }^{\mathrm{TM}}$ 588-A with and without DEAB, respectively. Approximately $10 \%$ of the cells showed high ALDH activity as indicated by using orange lines in the region gated by considering the results depicted in (B). 
(A)

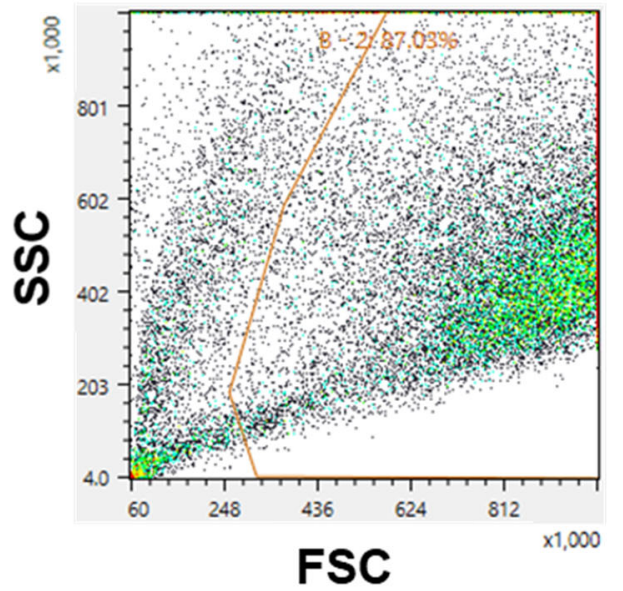

(C)

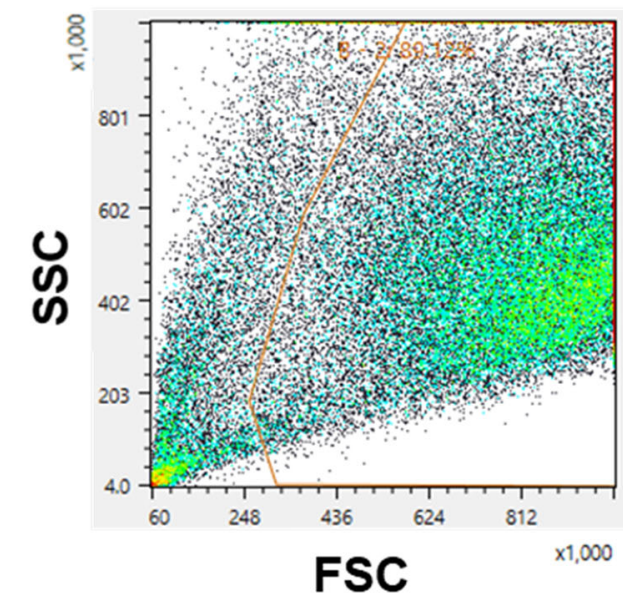

(B)

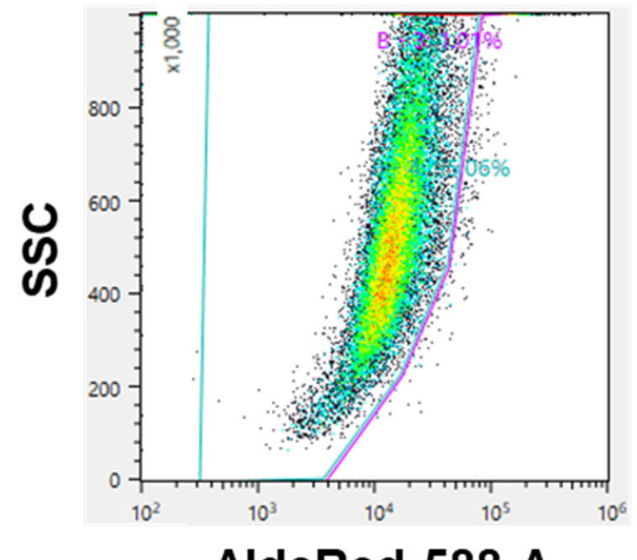

AldeRed 588-A

(D)

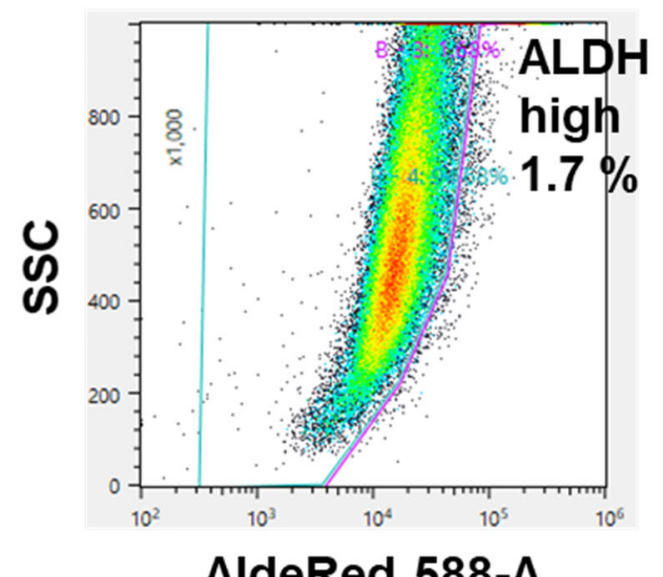

Figure S6. (A, B) and (C, D) show the results of flow cytometry analysis of MDA-MB-231 cells based on the ALDH activity assay with and without DEAB, respectively. Based on the intensities of FSC and SSC shown in Figures (A) and $(\mathrm{C})$, the gates were placed and the following fluorescence intensity analyses were performed for the gated cell population as indicated by using orange lines in Figures (A) and (C). Figures (B) and (D) show the fluorescence intensity of AldeRed ${ }^{\mathrm{TM}}$ 588-A with and without DEAB, respectively. Approximately $1 \%$ of the cells showed high ALDH activity as indicated by using purple lines in the region gated by considering the results depicted in (B). 
(A)

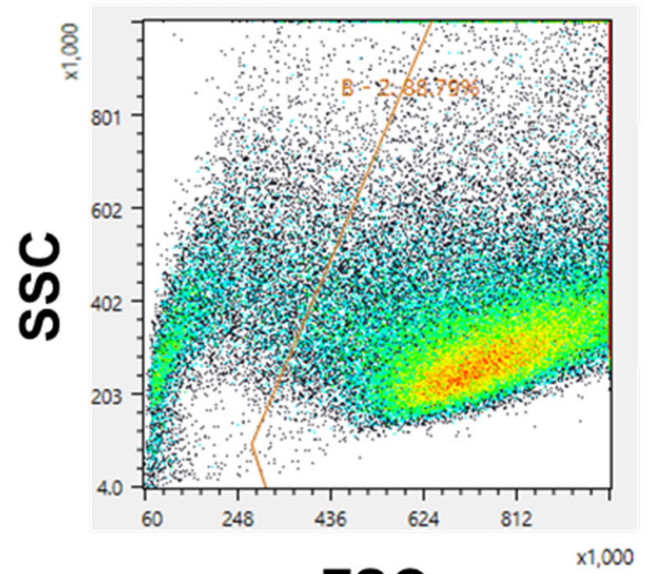

FSC
(B)

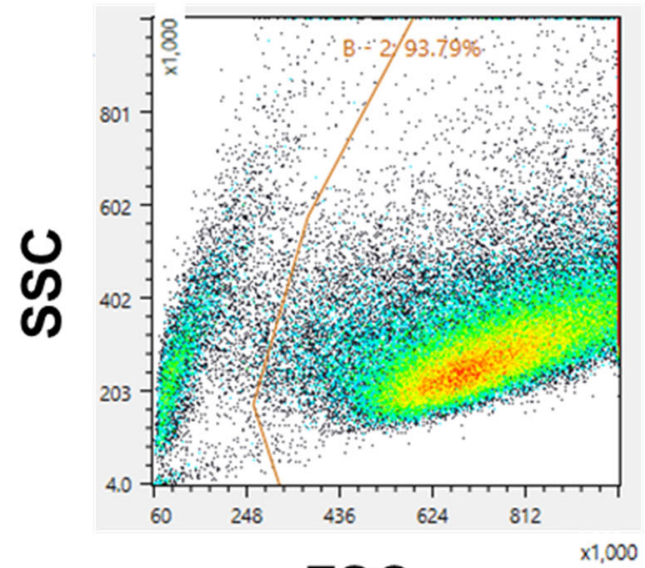

FSC

Figure S7. Flow cytometry analysis and sorting of HT29 cells using two types of solution, (A) RPMI-1640 medium and (B) AldeRed ${ }^{\mathrm{TM}}$ Assay Buffer. Since the small FSC population was hypothesized to contain dead cells, cell fragments, and dust, we set the gate based on the FSC intensity. The gated live cells were collected for the following viability and proliferation assays shown in Table S2 and Figure S8, respectively. 
(A)
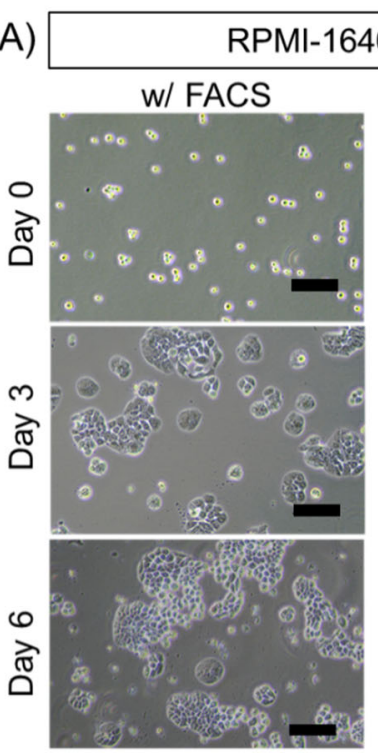

(B) w/o FACS
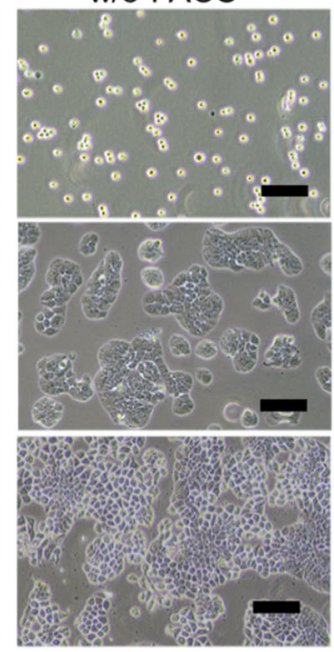
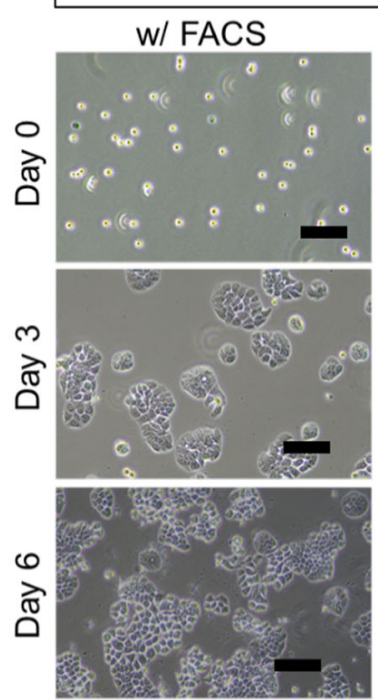

w/o FACS

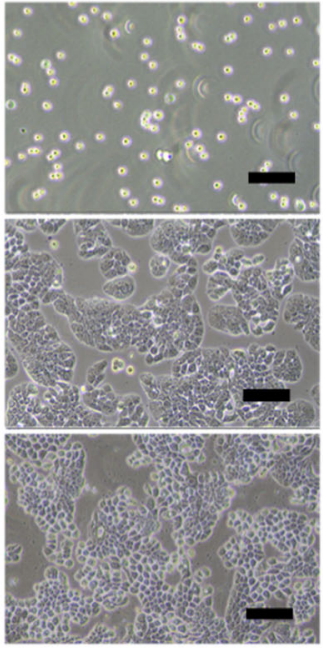

Figure S8. Typical cultured HT29 cell images acquired before and after FACS operation at Day 0 using different solutions, (A) RPMI-1640 medium and (B) AldeRed ${ }^{\mathrm{TM}}$ Assay Buffer, to verify the cell damages inflicted during the FACS operation. All scale bars are $100 \mu \mathrm{m}$. 
Table S1. Electrical conductivity of the buffer and medium used in this study.

\begin{tabular}{|c|c|}
\hline & Electrical conductivity (S/m) \\
\hline $1 \times$ PBS & $1.515 \pm 0.002$ \\
\hline RPMI & $1.159 \pm 0.002$ \\
\hline DMEM & $1.296 \pm 0.002$ \\
\hline EMEM & $1.287 \pm 0.001$ \\
\hline
\end{tabular}


Table S2. Table S2. A live/dead cell viability assay conducted via trypan blue staining performed before and after FACS operation in two types of solution, RPMI-1640 medium and AldeRed ${ }^{\mathrm{TM}}$ Assay Buffer.

\begin{tabular}{|c|c|c|c|c|}
\hline Solution & \multicolumn{2}{|c|}{ RPMI-1640 Medium } & \multicolumn{2}{c|}{ AldeRed ${ }^{\text {TM }}$ Assay Buffer } \\
\hline FACS operation & w/ FACS & w/o FACS & w/ FACS & w/o FACS \\
\hline $\begin{array}{c}\text { Cell viability } \\
\text { (\% of total cells) }\end{array}$ & $90.3 \%$ & $94.8 \%$ & $91.8 \%$ & $85.7 \%$ \\
\hline $\begin{array}{c}\text { Number of } \\
\text { measured cells }\end{array}$ & 154 & 575 & 159 & 391 \\
\hline
\end{tabular}

